\title{
MEASURING THERMAL CHARACTERISTICS OF THE HEAT EXCHANGER
}

\author{
Jindřich KŇOUREK, Michal KUS, Tomáš SYKA*
}

\begin{abstract}
New Technologies Research Centre at the University of West Bohemia in Pilsen uses middle-sized wind tunnel experimental facility. The tunnel equipment is extended in order to let us measure also heat transfer: the main wind tunnel duct itself forms the opened cooling air circuit, one closed circuit is there for water based coolant flow and one more closed circuit forms the hot compressed air loop. In this article, the typical measurement of the thermal characteristics of the common waterair heat exchanger is presented.
\end{abstract}

\section{Calorimetric Wind tunnel}

New Technologies Research Centre at the University of West Bohemia in Pilsen uses middle-sized wind tunnel experimental facility. You can see the wind tunnel configuration in the picture 1. Main parts are numbered as follows: Number 1 is the wind tunnel suction part, 2 is an axial fan. Number 3 is the duct reduction from the circular to the rectangular cross section. At the position of number 4 , there is a honeycomb structure placed in order to eliminate the non-axial flow velocities. Also some screen section is present there to ensure more uniform air flow profile in the tunnel. The wind tunnel continues by the duct section. Ducts are of rectangular profile $600 \mathrm{~mm} \times 495 \mathrm{~mm}$. Some duct sections are carried by wheeled supports (5) to make the wind tunnel configuration variable by changing required duct pieces.

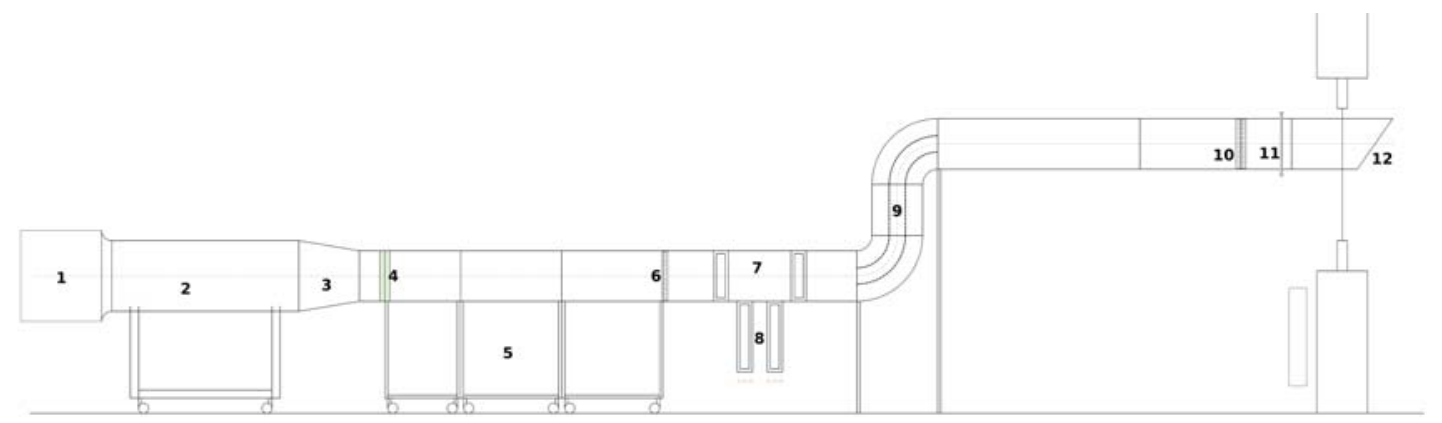

Figure 1: Wind tunnel configuration schema

There is one more section with honeycomb core in the tunnel (6). Number 7 stands for the measuring area where an investigated object is placed. In the planes in front of the object and behind the object, there are probes moving by the traversers (8). The tunnel continues by the double bended section 9 and some more straight duct pieces. At the position of number 10 there is another honeycomb core, 11 is the Wilson airflow grid [3]. Number 12 is the wind tunnel outlet. The air is sucked from within the laboratory and is blown out to the outside. The air way is about $15 \mathrm{~m}$ long.

This experimental facility can be used for different applications, e.g. velocity and pressure profiles measurements in front of and behind the investigated object, flow smoke-laser visualization, PIV measurements. But mostly, we use it for the calorimetric measurement of the heat exchangers of different types. The wind tunnel itself is an opened cooling air circuit. The facility is completed also with of one circuit of hot water based coolant circuit and one circuit for hot compressed air. In this article, the typical measurement of the thermal characteristics of the common water-air heat exchanger is presented.

\footnotetext{
* University of West Bohemia, New Technologies Research Centre, Univerzitni 8, CZ30614 Pilsen, Czech Republic http://www.ntc.zcu.cz, e-mail: knourek@ntc.zcu.cz
}

This is an Open Access article distributed under the terms of the Creative Commons Attribution License 2.0, which permits unrestricted use, distribution, and reproduction in any medium, provided the original work is properly cited. 


\section{CIRCUIT CHARACTERISTICS}

\subsection{COOLING AIR CIRCUIT}

The cooling air circuit is the wind tunnel itself, as it was described above. The axial fan has the nominal power of $15 \mathrm{~kW}$ and the idle mass flow rate $10 \mathrm{~m}^{3} / \mathrm{s}$. This is not much, but it is still covering typical operating points for automotive heat exchangers. This power allows us to obtain the air velocity up to $8 \mathrm{~m} / \mathrm{s}$ at the exchanger surface, depending on the pressure loss of the exchanger measured. In the car engine compartment, such an air velocity is also maximal one in common cases, so we can measure the exchanger properties under similar operating conditions. The mass flow rate in the circuit is set using the frequency changer of the axial fan $\mathrm{AC}$ motor.

\subsection{WATER-BASED COOLANT CIRCUIT}

The coolant circuit is closed loop with more than 80 liters of coolant. The main coolant circuit part is the pair of electrical boilers with heat power of $75 \mathrm{~kW}$ together. The heat power is regulated to achieve wanted heat power supplied to the coolant. We have two pumps in the system both controlled via frequency changer of the AC motors. We can choose a smaller pump, which is suited for lower mass flow rates of the coolant with higher pump head. The second one is for higher mass flow rates with lower pump head. The heated coolant is pumped via heat insulated pipes to the heat exchanger, which is mounted in the measurement area of the wind tunnel. In the circuit, we can set wanted overpressure to get similar conditions as in the car engine application. Also, we can control the coolant boiling point and avoid small bubble creation in the system.

\section{HEAT EXChANGER MEASUREMENTS}

The investigated heat exchanger is mounted in the wind tunnel in the measuring area. The heat exchanging part is filling up the cross section of the wind tunnel duct with no overlaps of missing areas. If necessary, the wind tunnel duct is modified to fulfill this condition. You can see mounted heat exchanger in the tunnel in the picture 2 .

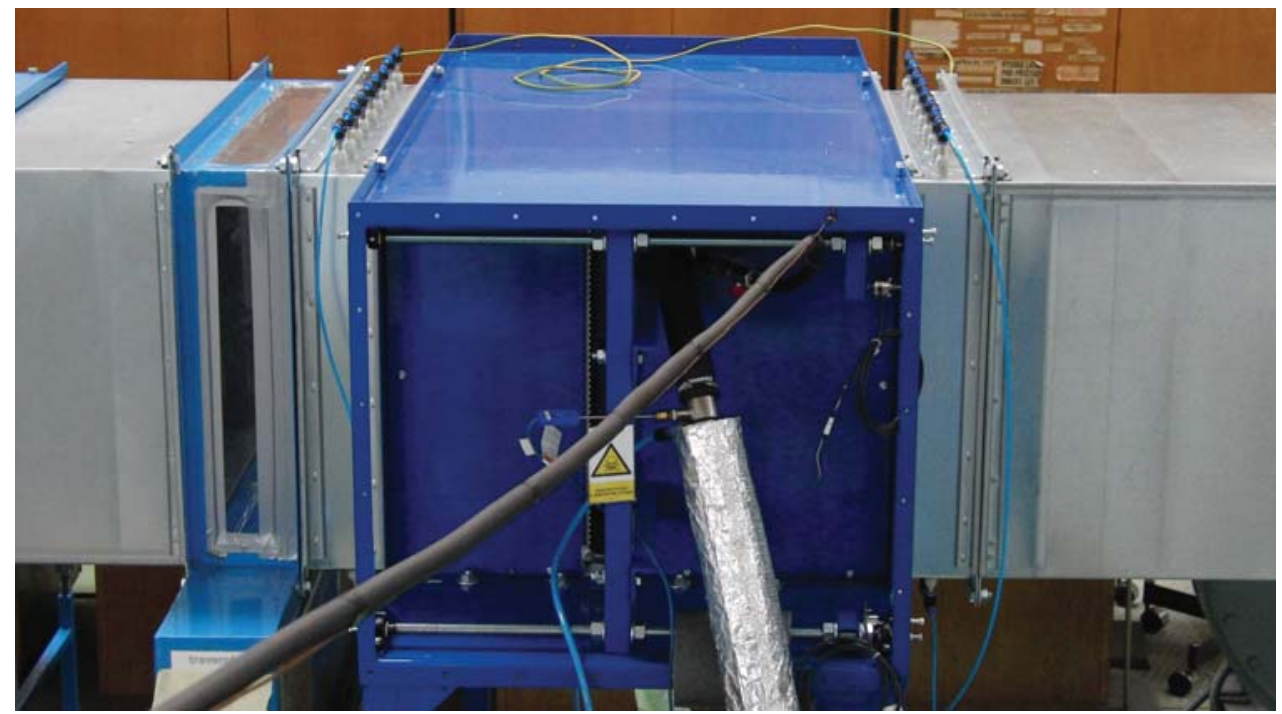

Figure 2: Measured heat exchanger in the measurement area

The air flow direction is from left to right, the glass window on the left side is there for us to be able to have a visible control of the probes moving in front of the heat exchanger. The investigated exchanger is mounted in the middle of the measuring area, you can see pipes junctions there. In planes in front of and behind the exchanger, there is a system of drilled pipes that serves for the measurement of the total press loss of the exchanger on the air side.

During the measurement, we want to achieve the balance of the heat power taken out by the cooling air and the heat power supplied into the coolant. This balance must be persisting in the system for several 
minutes to be easily measured. In addition, we want to keep the heat exchanger coolant inlet temperature at specified temperature and mass flow rate. The same is for cooling air, so the measurement is not easy in term of regulate all the quantities in reasonable limits to achieve accurate measurement results for given operating point.

At the wind tunnel side, quantities measured are: inlet and outlet air temperature meaning the average temperature in front of and behind the heat exchanger surface, mass flow rate, air pressure loss at the exchanger. At the coolant loop, the inlet and outlet temperature are measured, also mass flow rate and coolant pressure loss. From these values, we can derive the heat power exchanged.

\subsection{Measured Results}

Shown results are here presented for common automotive water-air heat exchanger. The heat characteristics are investigated for inlet air temperature at $25^{\circ} \mathrm{C}$. The coolant is mixture of unmineralized water and monoethylen-glycol in ratio 60:40, its exchanger inlet temperature is stabilized at $65^{\circ} \mathrm{C}$. Measurements are done for operating points with variation of coolant mass flow rate and also air mass flow rate.

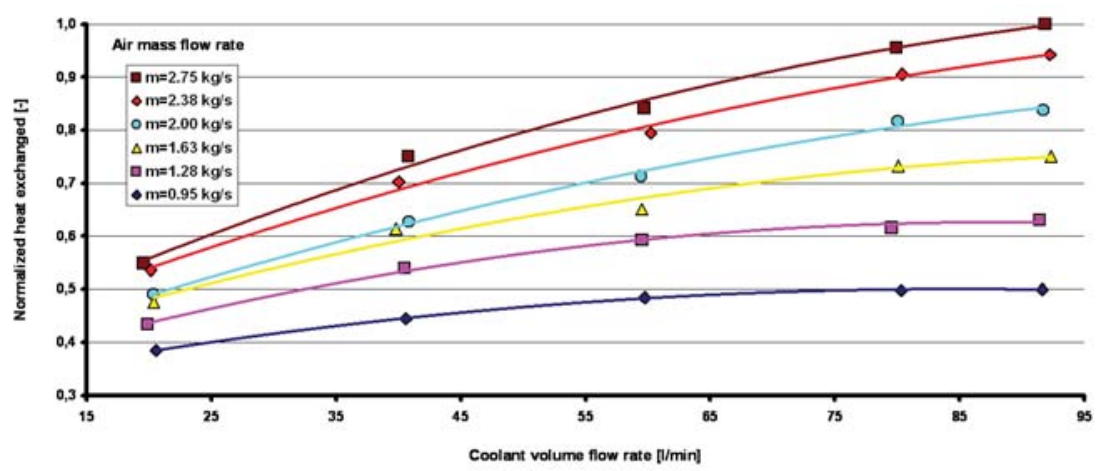

Figure 3: Normalized heat exchanged at the coolant side

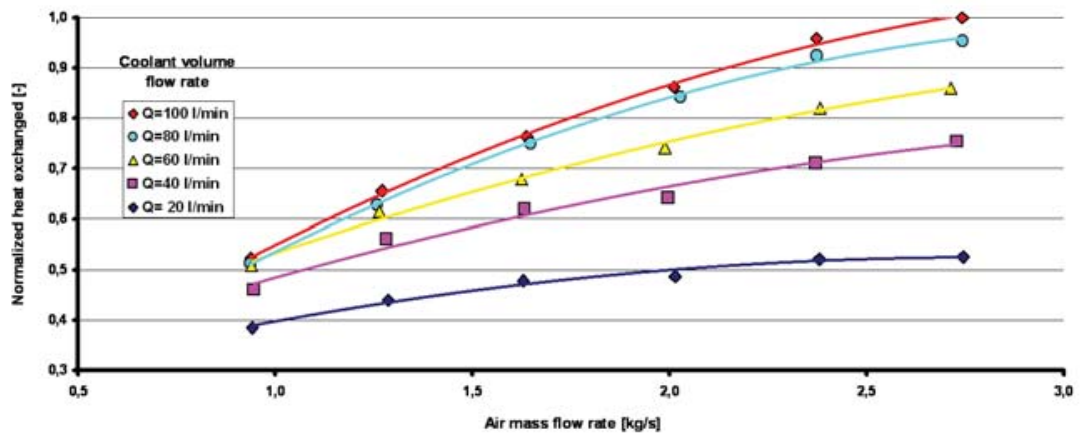

Figure 4: Normalized heat exchanged at the tunnel air side

In the graph at figure 3, there is the heat transferred from coolant to air depending on the coolant volume mass flow rate for different mass flow rates of cooling air. The heat is normalized meaning it is he ratio of the actual value measured to the maximum value measured. In the graph at figure 4, there is the normalized heat gained to air depending on the mass flow rate of air for different coolant volume flow rates. We measure the heat exchanged in both circuits separately. The heats measured are compared then. Both values should be the same, but because of some small inaccuracies during the measurements, they are not. Normally, the difference between both values are up to $3 \%$, and this means we are measuring well.

In the picture 5, there is the graph of efficiency depending on the coolant volume flow rate. In the picture 6 , there is the graph of normalized pressure loss of the heat exchanger at the air side. 


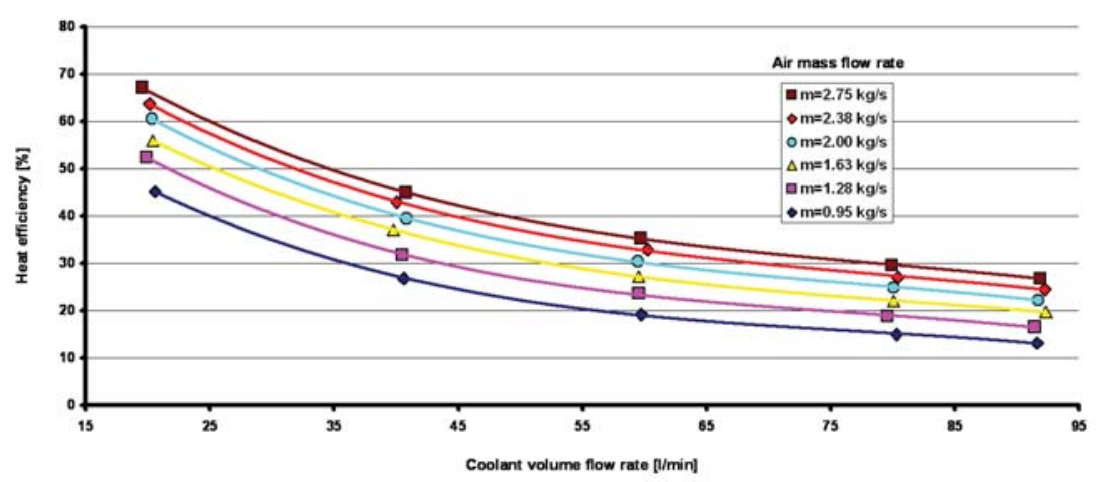

Figure 5: Heat transfer efficiency

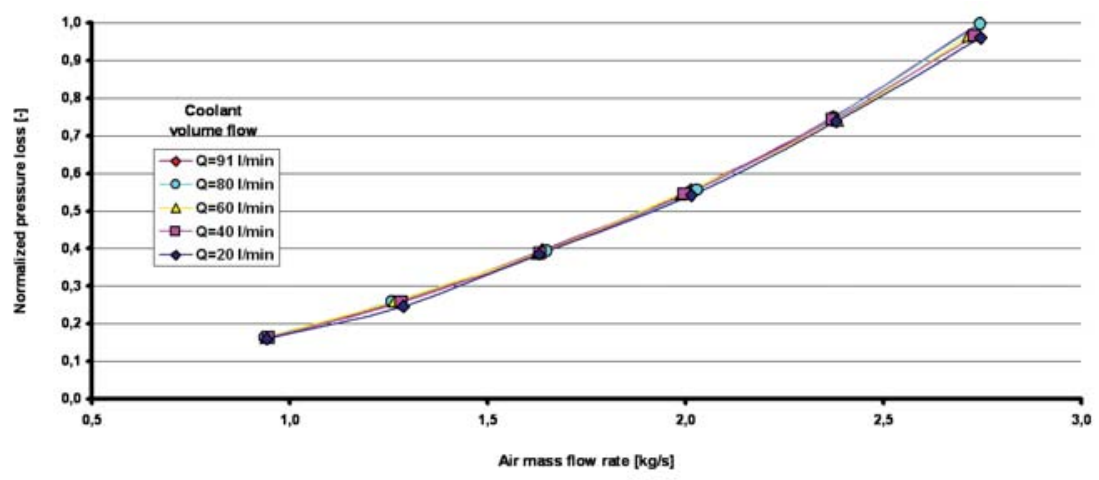

Figure 6: Exchanger pressure loss at the air side

\section{Conclusion}

Not only heat exchangers of water-air type can be measured. We are ready to measure the similar characteristics for the air-air heat exchanger using the compressed air circuit. We can also provide the measurement of the water-air type, meaning the cooled medium is hot air in this case and cooling medium is water-based liquid. Also, water-water exchangers can be measured. Moreover, we can investigate the behavior of the packet of two exchangers working simultaneously, the first one is waterair type and the second one is air-air type.

Spring of 2012 is an important milestone for our tunnel. We are moving the facility to new area. We will slightly upgrade the facility hardware and controlling software to get better behavior and higher parameters of the circuits. This will allow us to measure the heat exchanger in even better way.

\section{ACKNOWLEDGEMENT}

The result was developed within the CENTEM project, reg. no. CZ.1.05/2.1.00/03.0088 that is cofunded from the ERDF within the OP RDI programme of the Ministry of Education, Youth and Sports.

\section{REFERENCES}

[1] A Bejan and A D Kraus, Heat transfer handbook, John Wiley \& sons, Inc., 2003

[2] T Kuppan, Heat exchanger design handbook, Taylor \& Francis, 2000

[3] J Knourek and M Kus, Construction of home-made airflow Wilson grid and operational remarks, Proceedings of Experimental Fluid Mechanics 2010, 227-280, 2010 